\title{
Hidden Connections between Regression Models of Strain-Gage Balance Calibration Data
}

\author{
N. Ulbrich* \\ Jacobs Technology Inc., Moffett Field, California 94035-1000
}

\begin{abstract}
Hidden connections between regression models of wind tunnel strain-gage balance calibration data are investigated. These connections become visible whenever balance calibration data is supplied in its design format and both the Iterative and Non-Iterative Method are used to process the data. First, it is shown how the regression coefficients of the fitted balance loads of a force balance can be approximated by using the corresponding regression coefficients of the fitted strain-gage outputs. Then, data from the manual calibration of the Ames MK40 six-component force balance is chosen to illustrate how estimates of the regression coefficients of the fitted balance loads can be obtained from the regression coefficients of the fitted strain-gage outputs. The study illustrates that load predictions obtained by applying the Iterative or the Non-Iterative Method originate from two related regression solutions of the balance calibration data as long as balance loads are given in the design format of the balance, gage outputs behave highly linear, strict statistical quality metrics are used to assess regression models of the data, and regression model term combinations of the fitted loads and gage outputs can be obtained by a simple variable exchange.
\end{abstract}

\section{Nomenclature}

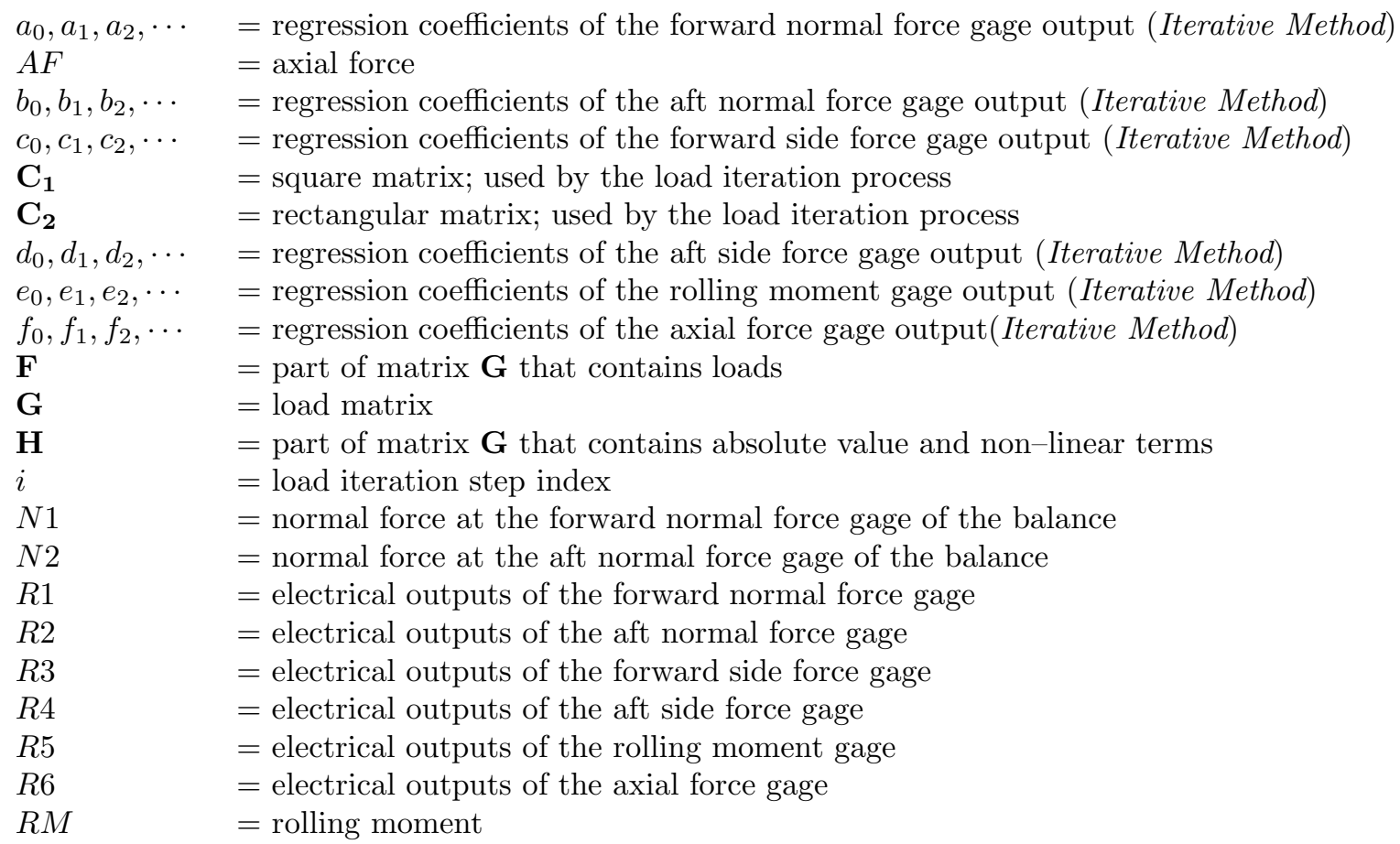

* Aerodynamicist, Jacobs Technology Inc. 
$\beta_{0}, \beta_{1}, \beta_{2}, \cdots=$ regression coefficients of the aft normal force (Non-Iterative Method)

$\gamma_{0}, \gamma_{1}, \gamma_{2}, \cdots=$ regression coefficients of the forward side force (Non-Iterative Method)

$\delta_{0}, \delta_{1}, \delta_{2}, \cdots=$ regression coefficients of the aft side force (Non-Iterative Method)

$\Delta \mathbf{R}=$ delta gage output vector or matrix

$\zeta_{0}, \zeta_{1}, \zeta_{2}, \cdots=$ regression coefficients of the axial force (Non-Iterative Method)

$\eta_{0}, \eta_{1}, \eta_{2}, \cdots=$ regression coefficients of the forward normal force (Non-Iterative Method)

$\mu_{0}, \mu_{1}, \mu_{2}, \cdots=$ regression coefficients of the rolling moment (Non-Iterative Method)

$\xi \quad=$ index of regression coefficient of a dominant regression model term

$\rho \quad=$ index of regression coefficient of a dominant regression model term

$\sigma \quad=$ index of regression coefficient of a dominant regression model term

$\phi \quad=$ index of regression coefficient of a dominant regression model term

$\psi \quad=$ index of regression coefficient of a dominant regression model term

$\omega \quad=$ index of regression coefficient of a dominant regression model term

\section{Introduction}

Different analysis approaches are used in the wind tunnel testing community to predict balance loads from measured strain-gage outputs during a wind tunnel test. One group of analysts, for example, processes balance calibration data by first fitting strain-gage outputs of the balance as a function of the applied balance loads. In that case, an iteration scheme is needed so that balance loads can be predicted from measured strain-gage outputs during a wind tunnel test. This analysis approach is called the Iterative Method (see Refs. [1], [2], and [3] for more detail).

In principle, the balance calibration experiment defines loads as independent variables and gage outputs as dependent variables. Some analysts prefer to switch the independent and dependent variables that the balance calibration experiment defines. This alternate analysis approach is called the Non-Iterative Method. In this case, no iteration is needed to predict loads from gage outputs during a wind tunnel test because the applied loads are directly fitted as a function of measured gage outputs (see Ref. [4] for more details).

Regression models used by the Iterative Method and Non-Iterative Method are derived from the same balance calibration data set. Therefore, in theory, they should contain the same information about the behavior of the balance even though, in one case, gage outputs are fitted as a function of balance loads and, in another case, balance loads are fitted as a function of gage outputs. The present paper studies the relationship between the regression models of the two fundamentally different analysis approaches in more detail as the balance characteristics themself must be contained in (i) math terms that are selected for the regression analysis and (ii) the sign and magnitude of the regression coefficients.

In general, it is recommended to process a balance calibration data set in its "design" load format. In other words - a force balance should be analyzed in force balance format, or, a moment balance should be analyzed in moment balance format. Then, the primary sensitivities of all gages of the balance exist (see Ref. [5] for more details). This characteristic also means that, in an ideal case, the gage outputs of single gage loadings are located along a straight line when plotted versus the corresponding single gage loads (a single gage loading is a setup during the calibration of a balance that applies a single load component to the balance while simultaneously keeping the magnitude of all other load components close to zero). The gage outputs of the remaining combined loadings will also be in the vicinity of this straight line.

Figure 1, for example, shows the output of the forward normal force gage of a force balance plotted versus the forward normal force. Both single gage loadings and combined loadings are depicted. It can be seen that the gage output is more or less proportional to the corresponding primary gage load. The required constant of proportionality is the inverse of the primary gage sensitivity of the gage. Therefore, a math model term combination selected to fit gage outputs as a function of balance loads could also be used to approximate the fit the corresponding primary gage load as a function of the gage outputs (and vice versa). It is only required to switch primary loads and gage outputs in the related regression models.

Now, the following question emerges: Can a direct connection between the coefficients used by the 
regression model of the Iterative and Non-Iterative Method be established if the primary loads and gage outputs are switched in the related regression models? Let us assume that the answer to the question is "yes." This means that the final balance load prediction accuracies of the Iterative and the Non-Iterative Method are more closely linked than a casual observer would suspect. It would also help users of the Iterative Method to gain confidence in the load prediction accuracy of the Non-Iterative Method (and vice versa).

First, in order to find an answer to the question posed in the previous paragraph, basic elements of the Iterative and Non-Iterative Method are reviewed for a typical force balance. Then, using a calibration data set of a force balance as an example, the connection between the regression coefficient sets will be investigated in more detail.

\section{Balance Calibration Data Analysis Methods}

\section{A. Iterative Method}

This section describes the balance calibration data analysis assuming the Iterative Method is applied to a force balance. Basic elements of the method are discussed in great detail in Ref. [1]. Therefore, only an abbreviated description of the application of the method to a force balance is given in this section. In principle, the Iterative Method is a two step process. First, gage outputs are fitted as a function of calibration loads. Then, the regression coefficients of the gage outputs are used to construct a load iteration process so that balance loads can be predicted from measured gage outputs during a wind tunnel test.

Data from the calibration of a force balance may be used to illustrate the application of the Iterative Method. It is assumed that (i) data of a six-component force balance is analyzed and that (ii) the loads are given in force balance format. Therefore, the regression models of the six gage outputs can be expressed as a function of the balance loads using the following equations:

$$
\begin{aligned}
& R 1=\underbrace{a_{0}}_{\text {intercept }}+\cdots+\underbrace{a_{\xi} \cdot N 1}_{\text {intercept }}+\cdots \\
& R 2=\underbrace{b_{0}}_{\text {dominant }}+\cdots+\underbrace{b_{0} \cdot N 2}_{\text {dominant }}+\cdots \\
& R 3=\underbrace{b_{0} \cdot N}_{\text {intercept }}+\cdots+\underbrace{d_{0} \cdot S 1}_{\text {dominant }}+\cdots \\
& R 4=\underbrace{e_{0}}_{\text {intercept }}+\cdots+\underbrace{c_{\text {dominant }}^{d_{\phi} \cdot S 2}}_{\text {intercept }}+\cdots \\
& R 5=\underbrace{e_{0} \cdot R M}_{\text {dominant }}+\cdots \\
& R 6=\underbrace{f_{\omega} \cdot A F}_{\text {dominant }}+\cdots
\end{aligned}
$$

The above equations highlight the fact that the regression model of each gage will be dominated by the influence of the primary gage load. Now, the six balance loads need to be computed iteratively after the completion of the regression analysis. The following iteration equation in combination with a load iteration process may be used for that purpose (see Ref. [1] for a description of the iteration process):

$$
F_{i}=\underbrace{\left[\mathrm{C}_{1}^{-1} \Delta \mathrm{R}\right]}_{\text {constant }}-\underbrace{\left[\mathrm{C}_{1}^{-1} \mathbf{C}_{2}\right] \cdot \mathbf{H}_{\mathrm{i}-1}}_{\text {changes for each iteration step }}
$$

Equation (2) is a matrix equation. Two matrices used in Eq. (2), i.e., $\mathbf{C}_{\mathbf{1}}$ and $\mathbf{C}_{\mathbf{2}}$, are derived from the regression coefficients of the gage outputs that are defined in Eqs. $(1 a)$ to $(1 f)$. The vector $\boldsymbol{\Delta} \mathbf{R}$ has 
the gage outputs that are measured when the balance experiences a load. Matrix $\mathbf{H}$ is constructed from the intermediate load estimates of the previous iteration step. The load iterations typically converge after 5 to 10 iterations assuming that a tolerance of $0.0001 \%$ of capacity is used to test for convergence.

\section{B. Non-Iterative Method}

The balance calibration data of a force balance may also be analyzed using the Non-Iterative Method. Differences between the Non-Iterative Method and the Iterative Method are discussed in great detail in Ref. [4]. Therefore, only basic elements of the Non-Iterative Method are reviewed in this section.

In principle, the Non-Iterative Method exchanges the independent and dependent variables that the Iterative Method uses. Now, gage outputs become independent variables and balance loads become dependent variables as far as the regression analysis of the balance calibration data is concerned. The Non-Iterative Method is a one step process as the loads are directly fitted as a function of the measured gage outputs. Consequently, no load iteration is required to predict loads from gage outputs during a wind tunnel test.

Again, data of a six-component force balance may be used to illustrate the application of the NonIterative Method. It is assumed that the calibration data of the force balance is given in force balance format. Then, we get the following six regression models for the analysis of the balance calibration data:

$$
\begin{aligned}
& N 1=\underbrace{\eta_{0}}_{\text {intercept }}+\cdots+\underbrace{\eta_{\xi} \cdot R 1}_{\text {dominant }}+\cdots \\
& N 2=\underbrace{\beta_{0}}_{\text {intercept }}+\cdots+\underbrace{\beta_{\rho} \cdot R 2}_{\text {dominant }}+\cdots \\
& S 1=\underbrace{\gamma_{0}}_{\text {intercept }}+\cdots+\underbrace{\gamma_{\sigma} \cdot R 3}_{\text {dominant }}+\cdots \\
& S 2=\underbrace{\delta_{0}}_{\text {intercept }}+\cdots+\underbrace{\delta_{\phi} \cdot R 4}_{\text {dominant }}+\cdots \\
& R M=\underbrace{\mu_{0}}_{\text {intercept }}+\cdots+\underbrace{\mu_{\psi} \cdot R 5}_{\text {dominant }}+\cdots \\
& A F=\underbrace{\zeta_{\omega} \cdot R 6}_{\text {dominant }}+\cdots
\end{aligned}
$$

The above equations highlight the fact that the regression model of each load component will be dominated by the primary gage output. Loads are computed during a wind tunnel test by using the measured gage outputs as input for the regression models of the loads that are defined in Eqs. $(3 a)$ to $(3 f)$.

In general, the Non-Iterative Method has the advantage that it is a one-step method. No iteration is needed to compute loads from measured strain-gage outputs during a wind tunnel test. An analyst, however, must not forget that the Non-Iterative Method ignores the fact that the balance loads are the "true" independent variables of the calibration experiment as loads are "applied" and strain-gage outputs are "measured" during the calibration of a balance. Therefore, the success of the Non-Iterative Method hinges on the fundamental assumption that a switch of the independent and dependent variables of the calibration data set does not negatively influence the mathematical description of the "true" physical behavior of the balance. In addition, the robustness and reliability of the regression model of each balance load depends on the fact that (i) the model does not have near-linear dependencies between terms and that (ii) it consists of statistically significant terms (see Ref. [6] for a discussion of these issues). These two requirements also apply to regression models of the gage outputs that the Iterative Method uses.

In the next section of the paper data from the calibration of NASA's MK40 balance is used to illustrate the connection between the regression coefficients of the Iterative Method and Non-Iterative Method. 


\section{Hidden Connection between Regression Models}

Data from the calibration of the NASA Ames MK40 force balance was chosen to illustrate the "hidden" connections that exist between the regression coefficients of the strain-gage outputs and the regression coefficients of the balance loads. The Ames MK40 balance was manufactured by the Task Corporation. It is a six-component force balance that measures five forces and one moment $(N 1, N 2, S 1, S 2, A F, R M)$. The balance has a diameter of 2.5 inches and a total length of 17.31 inches. Table 1 shows the load capacity of each load component.

Table 1: Load capacities of the NASA Ames 2.5in MK40 balance.

\begin{tabular}{|l|c|c|c|c|c|c|}
\hline & $N 1, l b s$ & $N 2, l b s$ & $S 1, l b s$ & $S 2, l b s$ & $R M$, in-lbs & $A F, l b s$ \\
\hline CAPACITY & 3500 & 3500 & 2500 & 2500 & 8000 & 400 \\
\hline
\end{tabular}

The original balance calibration was performed as a manual calibration. A total of 164 data points were taken in 16 load series. The analysis of the balance calibration data was done in several steps. First, the given calibration loads were tare corrected for the weight of the balance shell, calibration body, and other calibration fixtures. Then, the calibration data was analyzed using both the Iterative Method and the Non-Iterative Method that were described in previous sections.

For simplicity, it was decided to focus on hidden connections between regression coefficients of the forward normal force component and regression coefficients of the forward normal force gage output. Connections between regression coefficients of other load components and gage outputs can be investigated in a similar manner.

First, the Non-Iterative Method was applied to the calibration data of the MK40 balance. The analysis started by applying a regression model optimization process to the data so that the regression model of the forward normal force component would satisfy a set of widely accepted statistical quality requirements (see Refs. [7] and [8] for a description of the optimization process and the quality requirements). The optimization process chose the following 13-term regression model for the forward normal force component:

$$
\begin{aligned}
N 1= & \eta_{0}+\eta_{1} \cdot R 1+\eta_{2} \cdot R 2+\eta_{3} \cdot R 3+\eta_{4} \cdot R 5+\eta_{5} \cdot|R 1| \\
& +\eta_{6} \cdot|R 2|+\eta_{7} \cdot|R 3|+\eta_{8} \cdot|R 5|+\eta_{9} \cdot R 5 \cdot R 5 \\
& +\eta_{10} \cdot R 1 \cdot|R 1|+\eta_{11} \cdot R 2 \cdot|R 2|+\eta_{12} \cdot R 5 \cdot|R 5|
\end{aligned}
$$

The symbols $\eta_{0}, \eta_{1}, \ldots, \eta_{12}$ are the coefficients of the regression model of the forward normal force component. Figure 2a shows the Analysis of Variance result for the regression model of $N 1$. The math term and $t$-statistic columns are highlighted using blue rectangles. The $t$-statistic results confirm that the forward normal force gage output $R 1$ is the dominating term in the regression model of the forward normal force component $N 1$ as the term $R 1$ has the largest $t$-statistic magnitude $(+982)$. Figure $2 \mathrm{~b}$ shows the regression model and all coefficients of the forward normal force component. This is the regression model that the Non-Iterative Method ultimately uses for the prediction of the forward normal force component from the measured strain-gage outputs.

Now, the Iterative Method was applied to the calibration data of the MK40 balance. Similarly, the regression model optimization process identified the following 13-term regression model for the forward normal force gage output:

$$
\begin{aligned}
R 1= & a_{0}+a_{1} \cdot N 1+a_{2} \cdot N 2+a_{3} \cdot S 1+a_{4} \cdot R M+a_{5} \cdot|N 1| \\
& +a_{6} \cdot|N 2|+a_{7} \cdot|S 1|+a_{8} \cdot|R M|+a_{9} \cdot R M \cdot R M \\
& +a_{10} \cdot N 1 \cdot|N 1|+a_{11} \cdot N 2 \cdot|N 2|+a_{12} \cdot R M \cdot|R M|
\end{aligned}
$$

It is interesting to point out that the optimized regression model term combination listed on the right hand side of Eq. (5) can also be obtained by simply exchanging each primary gage output with the corresponding primary gage load in Eq. (4). The symbols $a_{0}, a_{1}, \ldots, a_{12}$ are the regression coefficients of the forward normal force gage output $R 1$. Figure 3a shows the Analysis of Variance result for the regression model of $R 1$. The math term and $t$-statistic columns are highlighted using blue rectangles. The $t$-statistic 
results confirm that the forward normal force component $N 1$ is the dominating term in the regression model of $R 1$ as the term $N 1$ has the largest $t$-statistic magnitude $(+891)$. Figure $3 \mathrm{~b}$ shows the optimized regression models and all coefficients of the six gage outputs of the MK40 balance. These coefficients are used to assemble the matrices $\mathbf{C}_{\mathbf{1}}$ and $\mathbf{C}_{\mathbf{2}}$ that the iteration equation and iteration process of the Iterative Method need for the prediction of balance loads from measured strain-gage outputs.

Now, a question comes up: Can the coefficients defined by the regression model of the forward normal force gage output $R 1$ (see Eq. (5)) be used to "reverse engineer" the coefficients of the regression model of the forward normal force component $N 1$ (see Eq. (4))? An answer to this question can be found by first solving the regression model of the forward normal force gage output $R 1$, i.e., Eq. (5), for the forward normal force component $N 1$. Then, after some algebra, we get the following equation from Eq. (5):

$$
\begin{aligned}
N 1= & \frac{-a_{0}}{a_{1}}+\frac{1}{a_{1}} \cdot R 1+\frac{-a_{2}}{a_{1}} \cdot N 2+\frac{-a_{3}}{a_{1}} \cdot S 1+\frac{-a_{4}}{a_{1}} \cdot R M+\frac{-a_{5}}{a_{1}} \cdot|N 1| \\
& +\frac{-a_{6}}{a_{1}} \cdot|N 2|+\frac{-a_{7}}{a_{1}} \cdot|S 1|+\frac{-a_{8}}{a_{1}} \cdot|R M|+\frac{-a_{9}}{a_{1}} \cdot R M \cdot R M \\
& +\frac{-a_{10}}{a_{1}} \cdot N 1 \cdot|N 1|+\frac{-a_{11}}{a_{1}} \cdot N 2 \cdot|N 2|+\frac{-a_{12}}{a_{1}} \cdot R M \cdot|R M|
\end{aligned}
$$

Unfortunately, Eq. (6) still has the loads $N 1, N 2, S 1$, and $R M$ on the right hand side of the equation. These loads, however, can be approximated by using the observations that (i) the original regression models of the strain-gage outputs each have a single dominant term and that (ii) all other terms of the regression model of the gage outputs are small when compared with the corresponding dominant term. Therefore, after simplifying Eqs. (1a), (1b), (1c), and (1e), and inspecting the coefficients given in Fig. 3b, we get:

$$
\begin{aligned}
& R 1 \approx a_{\xi} \cdot N 1 \quad \text { where } \xi=1 \\
& R 2 \approx b_{\rho} \cdot N 2 \quad \text { where } \rho=2 \\
& R 3 \approx c_{\sigma} \cdot S 1 \quad \text { where } \sigma=3 \\
& R 5 \approx e_{\psi} \cdot R M \quad \text { where } \psi=4
\end{aligned}
$$

The approximations given above can be solved for the four remaining load components that still need to be substituted on the right hand side of Eq. (6). Then, we get:

$$
\begin{array}{rlrlrl}
N 1 & \approx\left[1 / a_{1}\right] \cdot R 1 & \text { where } & {\left[1 / a_{1}\right]} & \equiv \text { primary sensitivity of } R 1 \\
N 2 \approx\left[1 / b_{2}\right] \cdot R 2 & \text { where } & {\left[1 / b_{2}\right]} & \equiv \text { primary sensitivity of } R 2 \\
S 1 & \approx\left[1 / c_{3}\right] \cdot R 3 & \text { where } & {\left[1 / c_{3}\right]} & \equiv \text { primary sensitivity of } R 3 \\
R M & \approx\left[1 / e_{4}\right] \cdot R 5 & \text { where } & {\left[1 / e_{4}\right]} & \equiv \text { primary sensitivity of } R 5
\end{array}
$$

Finally, after using Eqs. $(8 a)$ to $(8 d)$ to subsitute the remaining load components on the right hand side of Eq. (6) and after some algebra, we get the following approximation of the regression model of the forward normal force component $N 1$ of the MK40 balance:

$$
\begin{aligned}
N 1 \approx & \frac{-a_{0}}{a_{1}}+\frac{1}{a_{1}} \cdot R 1+\frac{-a_{2}}{a_{1} b_{2}} \cdot R 2+\frac{-a_{3}}{a_{1} c_{3}} \cdot R 3+\frac{-a_{4}}{a_{1} e_{4}} \cdot R 5+\frac{-a_{5}}{a_{1}\left|a_{1}\right|} \cdot|R 1| \\
& +\frac{-a_{6}}{a_{1}\left|b_{2}\right|} \cdot|R 2|+\frac{-a_{7}}{a_{1}\left|c_{3}\right|} \cdot|R 3|+\frac{-a_{8}}{a_{1}\left|e_{4}\right|} \cdot|R 5|+\frac{-a_{9}}{a_{1} e_{4} e_{4}} \cdot R 5 \cdot R 5 \\
& +\frac{-a_{10}}{a_{1} a_{1}\left|a_{1}\right|} \cdot R 1 \cdot|R 1|+\frac{-a_{11}}{a_{1} b_{2}\left|b_{2}\right|} \cdot R 2 \cdot|R 2|+\frac{-a_{12}}{a_{1} e_{4}\left|e_{4}\right|} \cdot R 5 \cdot|R 5|
\end{aligned}
$$


The exact solutions of the regression coefficients of the forward normal force component $N 1$ are defined in Eq. (4) and listed in Fig. 2b. These coefficients, i.e., ...

$$
\eta_{0}, \eta_{1}, \eta_{2}, \eta_{3}, \eta_{4}, \cdots
$$

may be compared with the "reverse engineered" approximations that were obtained from the regression model of gage output $R 1$. They are defined in Eq. (9) as follows:

$$
\frac{-a_{0}}{a_{1}}, \frac{1}{a_{1}}, \frac{-a_{2}}{a_{1} b_{2}}, \frac{-a_{3}}{a_{1} c_{3}}, \frac{-a_{4}}{a_{1} e_{4}}, \cdots
$$

The regression coefficients of the fitted gage outputs are listed in Fig. 3b. Therefore, it is possible to compute the "reverse engineered" approximations of the regression coefficients of the forward normal force and compare them with the exact solutions that are depicted in Fig. 2b.

The table in Fig. 4 shows the result of the comparison of the exact and approximated coefficient sets. Two observations can be made after inspecting the table: (i) signs of the exact and approximated coefficients match; (ii) magnitudes of the exact and approximated coefficients show good agreement.

The influence of each individual coefficient on the regression model was also investigated in more detail. Therefore, the exact solution was modified by replacing one coefficient at a time by its approximation. Then, the standard deviation of the load residuals was computed for the modified regression model that consisted of one approximated and twelve exact coefficients. The computed standard deviations are shown in the last column of Fig. 4. We observe that the largest standard deviation is reported for the case when the coefficient of the most significant term, indicated by the $t$-statistic of +982 , is replaced by its approximation. This observation is expected as the standard deviation of the load residuals must reach a maximum when the most significant coefficient is replaced by its approximation.

Finally, it is of interest to compare the standard deviation of the residuals of the forward normal force component for the three different analysis options that are discussed in the present paper. Table 2 below lists the standard deviation as a percentage of the capacity of the forward normal force component.

Table 2: Standard deviation of the load residuals of the forward normal force $N 1$.

\begin{tabular}{|c|c|}
\hline BALANCE CALIBRATION DATA ANALYSIS APPROACH & STANDARD DEVIATION \\
\hline ITERATIVE METHOD $\Longleftrightarrow$ exact solution, Eq. (2) & $0.0515 \%$ CAP. \\
\hline NON-ITERATIVE METHOD $\Longleftrightarrow$ exact solution, Eq. (4) & $0.0489 \%$ CAP. \\
\hline NON-ITERATIVE METHOD $\Longleftrightarrow$ approximation, Eq. (9) & $0.0646 \%$ CAP. \\
\hline
\end{tabular}

As expected, the standard deviations of the exact solution for the Iterative Method and the exact solution of the Non-Iterative Method show excellent agreement as the difference between the two standard deviations is only $0.0026 \%$. The standard deviation of the approximation of the Non-Iterative Method shows reasonable agreement with the corresponding exact solution as the difference is $0.0157 \%$. At this point it is important to emphasize that the approximation of the Non-Iterative Method should never be used instead of the corresponding exact solution for the calculation of balance loads. The approximation was only developed for the present study to show that a "hidden" connection between the regression coefficients of the Iterative and Non-Iterative Method can be established.

\section{Conclusions}

The present study illustrates that "hidden" connections between regression coefficient sets used by the Iterative and Non-Iterative Method may exist. It is possible to estimate sign and magnitude of the regression coefficients of the fitted loads by using the regression coefficients of the fitted gage outputs as long as (i) balance data is analyzed in its design format, (ii) the regression models of the loads and gage outputs meet rigorous statistical quality requirements, and (iii) the regression model terms can be obtained by simply switching primary loads and gage outputs. Numerical differences between the exact and approximated 
regression coefficient sets remain. They reflect the fact that the regression models used by the Iterative and Non-Iterative Method are still independent regression solutions of a given balance calibration data set.

Results of the present study may help users of both the Iterative and Non-Iterative Method to better understand how the two balance calibration data analysis approaches are connected to each other. It must also not be forgotten that the application of the Non-Iterative Method requires a switch of the independent and dependent variables that the balance calibration experiment defines. Questions about the validity of this variable exchange may come up at some point in time. Then, the approach used in the present investigations could be used to assess the influence of this variable exchange on the balance load estimates that are obtained by applying the Non-Iterative Method.

\section{Acknowledgements}

The author would like to thank Tom Volden of Jacobs Technology for his critical and constructive review of the final manuscript. The work reported in this paper was supported by the Wind Tunnel Division at NASA Ames Research Center under contract NNA09DB39C.

\section{References}

${ }^{1}$ AIAA/GTTC Internal Balance Technology Working Group, "Recommended Practice, Calibration and Use of Internal Strain-Gage Balances with Application to Wind Tunnel Testing," AIAA R-091-2003, American Institute of Aeronautics and Astronautics, Reston, Virginia, 2003.

${ }^{2}$ Ulbrich, N. and Volden, T., "Strain-Gage Balance Calibration Analysis Using Automatically Selected Math Models," AIAA 2005-4084, paper presented at the 41st AIAA/ASME/SAE/ASEE Joint Propulsion Conference and Exhibit, Tucson, Arizona, July 2005.

${ }^{3}$ Ulbrich, N. and Volden, T., "Application of a New Calibration Analysis Process to the MK-III-C Balance," AIAA 2006-0517, paper presented at the 44th AIAA Aerospace Sciences Meeting and Exhibit, Reno, Nevada, January 2006.

${ }^{4}$ Ulbrich, N., "Comparison of Iterative and Non-Iterative Strain-Gage Balance Load Calculation Methods," AIAA 2010-4202, paper presented at the 27th AIAA Aerodynamic Measurement Technology and Ground Testing Conference, Chicago, Illinois, June/July 2010.

${ }^{5}$ Ulbrich, N., "Influence of Primary Gage Sensitivities on the Convergence of Balance Load Iterations," AIAA 2012-0322, paper presented at the 50th AIAA Aerospace Sciences Meeting and Exhibit, Nashville, Tennessee, January 2012.

${ }^{6}$ Ulbrich, N. and Volden, T. "Regression Model Term Selection for the Analysis of Strain-Gage Balance Calibration Data," AIAA 2010-4545, paper presented at the 27th AIAA Aerodynamic Measurement Technology and Ground Testing Conference, Chicago, Illinois, June/July 2010.

${ }^{7}$ Ulbrich, N. and Volden, T., "Regression Analysis of Experimental Data Using an Improved Math Model Search Algorithm," AIAA 2008-0833, paper presented at the 46th AIAA Aerospace Sciences Meeting and Exhibit, Reno, Nevada, January 2008.

${ }^{8}$ Ulbrich, N., "Regression Model Optimization for the Analysis of Experimental Data," AIAA 2009-1344, paper presented at the 47th AIAA Aerospace Sciences Meeting and Exhibit, Orlando, Florida, January 2009. 


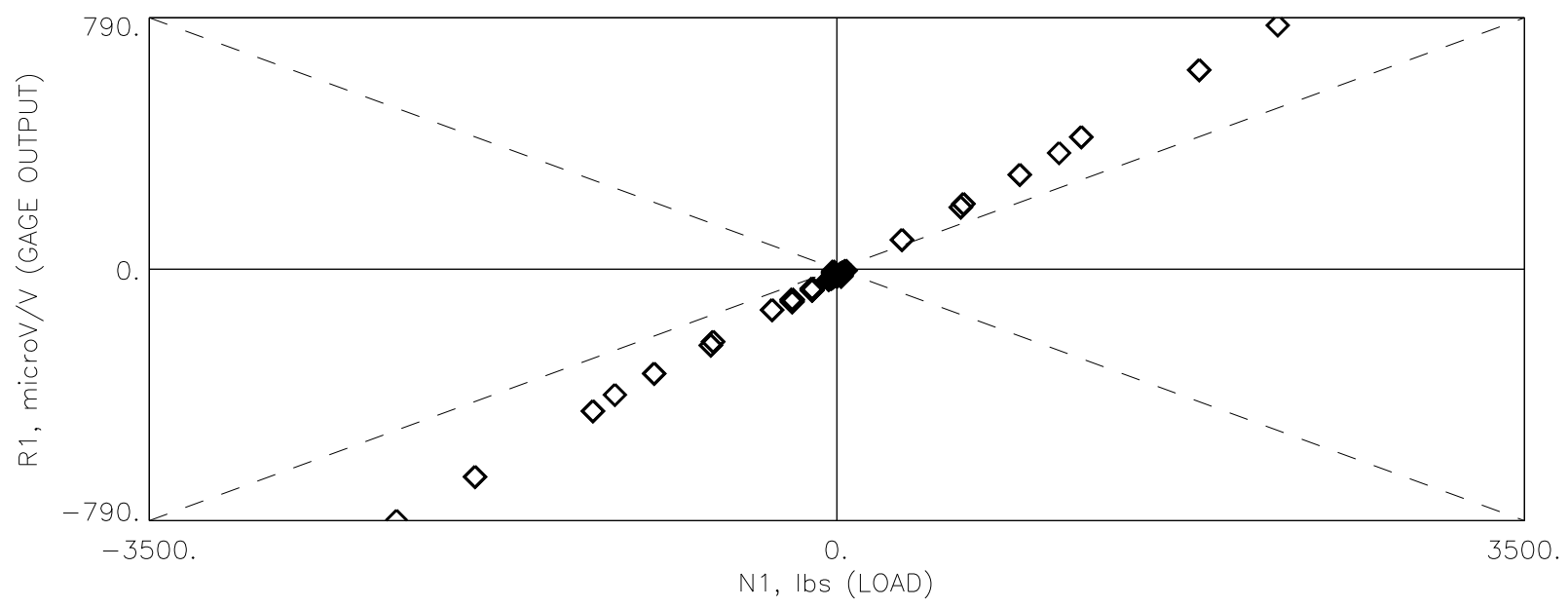

Fig. 1 Relationship between primary gage output $R 1$ and tare corrected primary gage load $N 1$.

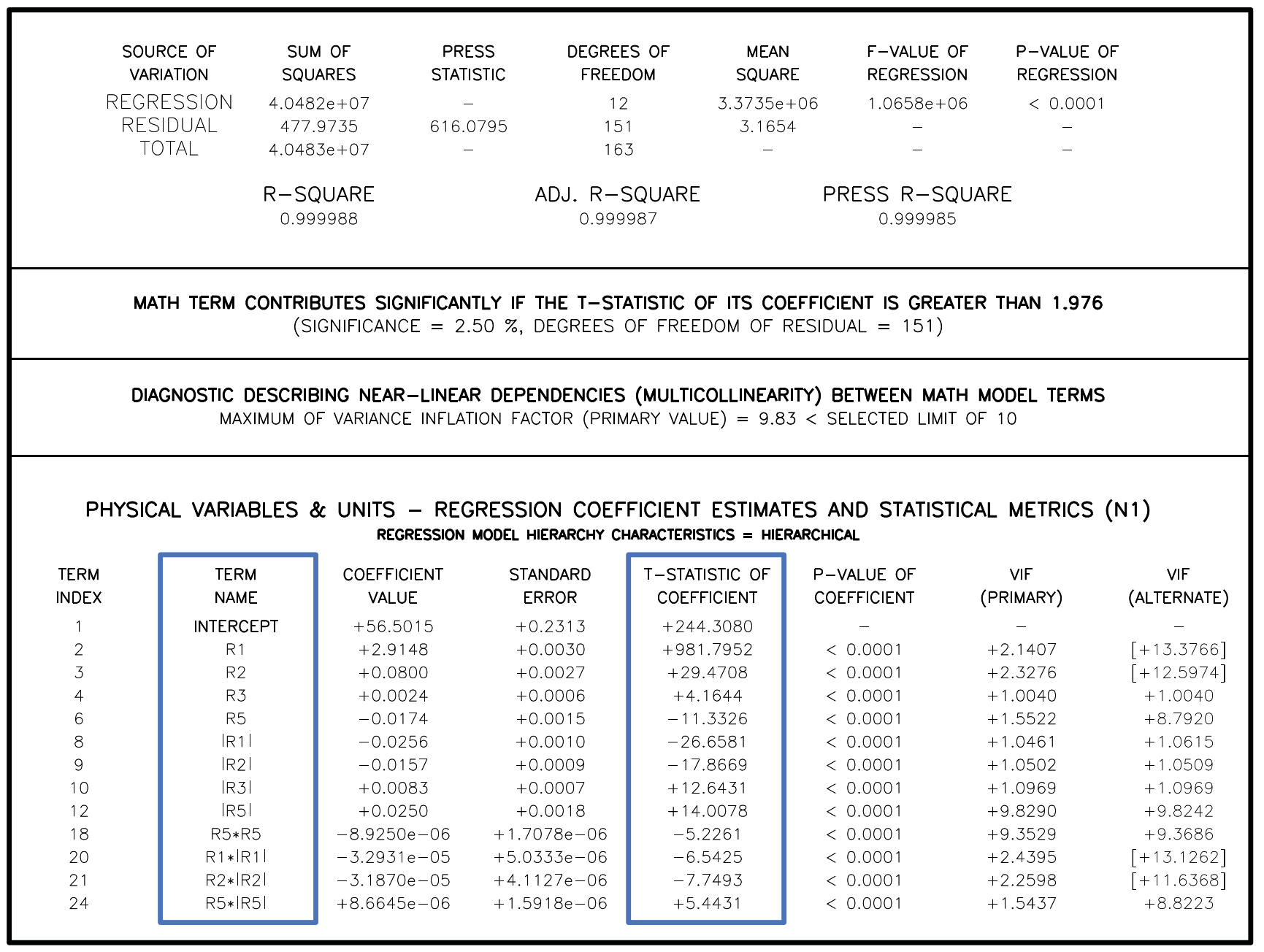

Fig. 2a Non-Iterative Method: Analysis of Variance results for regression model of forward normal force $N 1$.

American Institute of Aeronautics and Astronautics 


\begin{tabular}{|c|c|c|c|}
\hline INDEX & TERM & & N1 \\
\hline 1 & INTERCEPT & $\eta_{0}=$ & $+5.650150 e+01$ \\
\hline 2 & R1 & $\eta_{1}=$ & $+2.914783 e+00$ \\
\hline 3 & $\mathrm{R} 2$ & $\eta_{2}=$ & $+7.997201 e-02$ \\
\hline 4 & R3 & $\eta_{3}=$ & $+2.409364 \mathrm{e}-03$ \\
\hline 6 & R5 & $\eta_{4}=$ & $-1.741967 e-02$ \\
\hline 8 & IR1I & $\eta_{5}=$ & $-2.560737 e-02$ \\
\hline 9 & |R2| & $\eta_{6}=$ & $-1.566803 e-02$ \\
\hline 10 & |R3| & $\eta_{7}=$ & $+8.287214 e-03$ \\
\hline 12 & |R5| & $\eta_{8}=$ & $+2.498232 e-02$ \\
\hline 18 & $\mathrm{R} 5 * \mathrm{R} 5$ & $\eta_{9}=$ & $-8.924993 e-06$ \\
\hline 20 & $\mathrm{R} 1 *|\mathrm{R} 1|$ & $\eta_{10}=$ & $-3.293052 e-05$ \\
\hline 21 & $\mathrm{R} 2 *|\mathrm{R} 2|$ & $\eta_{11}=$ & $-3.187047 e-05$ \\
\hline 24 & $\mathrm{R} 5 *|\mathrm{R} 5|$ & $\eta_{12}=$ & $+8.664467 e-06$ \\
\hline
\end{tabular}

Fig. 2b Non-Iterative Method: Coefficients of optimized regression model of forward normal force $N 1$.

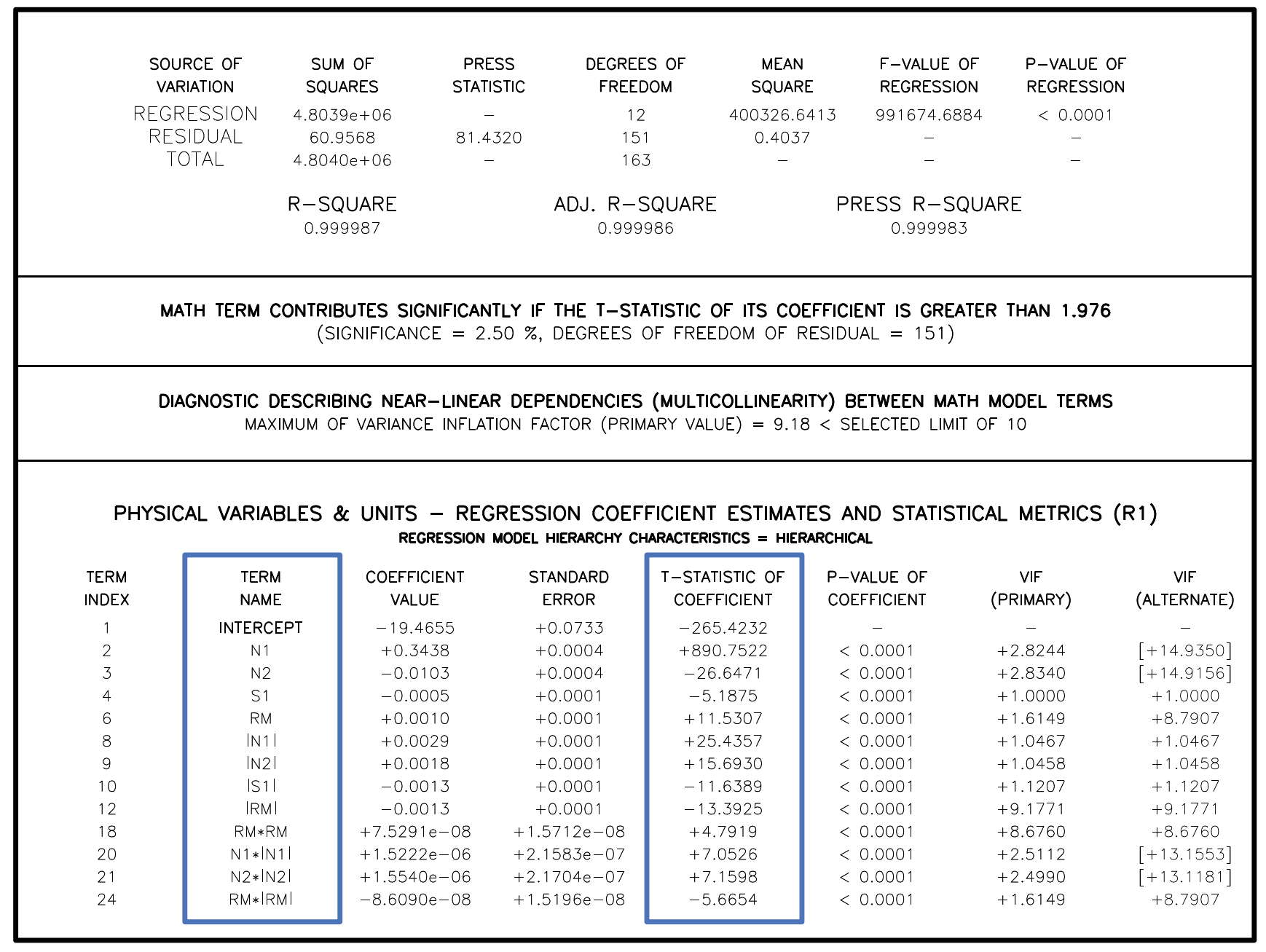

Fig. 3a Iterative Method: Analysis of Variance results for regression model of gage output $R 1$. 


\begin{tabular}{|c|c|c|c|c|c|c|c|}
\hline INDEX & TERM & R1 & R2 $\quad \mathbf{b}$ & R3 & R4 & R5 & R6 \\
\hline 1 & INTERCEPT $\mathrm{a}_{0}=$ & $-1.946546 e+01$ & $-2.116026 e+0$ & $-1.571845 e+0$ & $-5.743596 e+01$ & $-1.976257 e+0$ & $-1.384679 e+02$ \\
\hline 2 & $\mathrm{~N} 1 \mathrm{a}_{1} \Rightarrow>$ & $+3.437529 \mathrm{e}-01$ & $-4.302222 \mathrm{e}-1 / 2$ & $+6.338849 e-\beta 3$ & 0 & 0 & $+4.835826 \mathrm{e}-03$ \\
\hline 3 & N2 $\quad a_{2}=$ & $-1.032882 e-02$ & $+3.725837 \mathrm{e}-01$ & $+1.123877 e^{d} / 03$ & $+3.857918 \mathrm{e}-03$ & $-4.199624 e-34$ & $-2.833567 e-03$ \\
\hline 4 & $a_{3}=$ & $-5.235353 e-04$ & $+2.862075 e-03$ & $+4.905652 \mathrm{e}-01$ & $-2.170326 e-02$ & $+4.852466 \mathrm{e}-04$ & $-2.901236 e-03$ \\
\hline 5 & S2 & 0 & $+2.409149 \mathrm{e}-03$ & $-6.977922 \mathrm{e}-03$ & $+5.014106 \mathrm{e}-01$ & $-3.528204 e^{N} / 04$ & $+5.446800 e-03$ \\
\hline 6 & $a_{4}=$ & $+1.035526 e-03$ & $-4.833653 e-04$ & $+4.792302 \mathrm{e}-03$ & $+5.291577 \mathrm{e}-03$ & $+1.638645 e-01$ & $+2.991912 \mathrm{e}-03$ \\
\hline 7 & $\mathrm{AF}$ & 0 & $-2.708377 e-03$ & 0 & 0 & $+1.310120 \mathrm{e}-02$ & $+3.569037 e+00$ \\
\hline 8 & IN1| & $+2.888430 \mathrm{e}-03$ & $+1.458110 \mathrm{e}-03$ & $-2.173966 \mathrm{e}-03$ & 0 & $+3.078684 \mathrm{e}-04$ & $+1.276945 e-03$ \\
\hline 9 & $a_{6}=$ & $+1.786223 e-03$ & $+4.314710 \mathrm{e}-03$ & 0 & $-1.371239 e-03$ & 0 & $+1.440165 e-03$ \\
\hline 10 & $a_{7}=$ & $-1.340216 \mathrm{e}-03$ & $-6.562695 e-04$ & $+1.105774 \mathrm{e}-02$ & $+1.303598 \mathrm{e}-03$ & 0 & 0 \\
\hline 11 & IS2| & 0 & $-7.611073 e-04$ & $+1.495335 \mathrm{e}-03$ & $+1.321102 \mathrm{e}-02$ & 0 & 0 \\
\hline 12 & IRMI & $-1.316052 \mathrm{e}-03$ & $-1.115338 \mathrm{e}-03$ & $+3.617666 \mathrm{e}-03$ & $+1.803646 \mathrm{e}-03$ & $+1.155335 e-04$ & 0 \\
\hline 13 & $|A F|$ & 0 & 0 & 0 & 0 & 0 & 0 \\
\hline 14 & $\mathrm{~N} 1 * \mathrm{~N} 1$ & 0 & 0 & 0 & 0 & 0 & 0 \\
\hline 15 & $\mathrm{~N} 2 * \mathrm{~N} 2$ & 0 & 0 & 0 & 0 & 0 & 0 \\
\hline 16 & $\mathrm{~S} 1 * \mathrm{~S} 1$ & 0 & 0 & 0 & 0 & 0 & 0 \\
\hline 17 & $\mathrm{~S} 2 * \mathrm{~S} 2$ & 0 & 0 & 0 & 0 & 0 & $-9.028354 e-07$ \\
\hline 18 & $R M * R M$ & $+7.529071 \mathrm{e}-08$ & $+5.546812 \mathrm{e}-08$ & $-3.001823 e-07$ & $-1.347943 e-07$ & 0 & $+2.522531 \mathrm{e}-07$ \\
\hline 19 & $\mathrm{AF} * \mathrm{AF}$ & 0 & 0 & 0 & 0 & 0 & 0 \\
\hline 20 & $\mathrm{~N} 1 *|\mathrm{~N} 1|$ & $+1.522183 e-06$ & $+1.368479 e-06$ & 0 & 0 & 0 & 0 \\
\hline 21 & $\mathrm{~N} 2 *|\mathrm{~N} 2|$ & $+1.553984 \mathrm{e}-06$ & $+1.580928 \mathrm{e}-06$ & 0 & $-1.270365 \mathrm{e}-06$ & 0 & 0 \\
\hline 22 & $S 1 *|S 1|$ & 0 & 0 & 0 & 0 & 0 & 0 \\
\hline 23 & $S 2 * \mid S 21$ & 0 & $-8.325298 \mathrm{e}-07$ & 0 & $+1.574352 \mathrm{e}-06$ & 0 & 0 \\
\hline 24 & $\mathrm{RM} *|\mathrm{RM}|$ & $-8.609022 e-08$ & 0 & $-1.771749 e-07$ & $-1.772552 e-07$ & $-4.532282 e-08$ & 0 \\
\hline 25 & $A F *|A F|$ & 0 & 0 & 0 & 0 & 0 & 0 \\
\hline 26 & $\mathrm{~N} 1 * \mathrm{~N} 2$ & 0 & $-1.739066 \mathrm{e}-06$ & 0 & 0 & 0 & 0 \\
\hline 27 & $\mathrm{~N} 1 * \mathrm{~S} 1$ & 0 & 0 & 0 & 0 & 0 & 0 \\
\hline 28 & $\mathrm{~N} 1 * \mathrm{~S} 2$ & 0 & 0 & 0 & 0 & 0 & 0 \\
\hline 29 & $\mathrm{~N} 1 * \mathrm{RM}$ & 0 & 0 & 0 & 0 & 0 & 0 \\
\hline 30 & $N 1 * A F$ & 0 & 0 & 0 & 0 & 0 & 0 \\
\hline 31 & $\mathrm{~N} 2 * \mathrm{~S} 1$ & 0 & 0 & 0 & 0 & 0 & 0 \\
\hline 32 & $\mathrm{~N} 2 * \mathrm{~S} 2$ & 0 & 0 & 0 & 0 & 0 & 0 \\
\hline 33 & $\mathrm{~N} 2 * \mathrm{RM}$ & 0 & 0 & 0 & 0 & 0 & 0 \\
\hline 34 & $\mathrm{~N} 2 * \mathrm{AF}$ & 0 & 0 & 0 & 0 & 0 & 0 \\
\hline 35 & $\mathrm{~S} 1 * \mathrm{~S} 2$ & 0 & 0 & 0 & 0 & 0 & 0 \\
\hline 36 & $\mathrm{~S} 1 * \mathrm{RM}$ & 0 & 0 & 0 & 0 & 0 & 0 \\
\hline 37 & $\mathrm{~S} 1 * \mathrm{AF}$ & 0 & 0 & 0 & 0 & 0 & 0 \\
\hline 38 & $\mathrm{~S} 2 * \mathrm{RM}$ & 0 & 0 & 0 & 0 & 0 & 0 \\
\hline 39 & $\mathrm{~S} 2 * \mathrm{AF}$ & 0 & 0 & 0 & 0 & 0 & 0 \\
\hline 40 & $\mathrm{RM} * \mathrm{AF}$ & 0 & 0 & 0 & 0 & 0 & 0 \\
\hline 41 & $|\mathrm{~N} 1 * \mathrm{~N} 2|$ & 0 & 0 & 0 & 0 & 0 & 0 \\
\hline 42 & $|\mathrm{~N} 1 * \mathrm{~S} 1|$ & 0 & 0 & 0 & 0 & 0 & 0 \\
\hline 43 & $|\mathrm{~N} 1 * \mathrm{~S} 2|$ & 0 & 0 & 0 & 0 & 0 & 0 \\
\hline 44 & $|\mathrm{~N} 1 * \mathrm{RM}|$ & 0 & 0 & 0 & 0 & 0 & 0 \\
\hline 45 & $|\mathrm{~N} 1 * \mathrm{AF}|$ & 0 & 0 & 0 & 0 & 0 & 0 \\
\hline 46 & IN2*S1| & 0 & 0 & 0 & 0 & 0 & 0 \\
\hline 47 & $|\mathrm{~N} 2 * \mathrm{~S} 2|$ & 0 & 0 & 0 & 0 & 0 & 0 \\
\hline 48 & |N2*RM| & 0 & 0 & 0 & 0 & 0 & 0 \\
\hline 49 & $|\mathrm{~N} 2 * \mathrm{AF}|$ & 0 & 0 & 0 & 0 & 0 & 0 \\
\hline 50 & $|\mathrm{~S} 1 * \mathrm{~S} 2|$ & 0 & 0 & 0 & 0 & 0 & 0 \\
\hline \multicolumn{8}{|c|}{ (only 50 of 97 regression coefficient rows shown) } \\
\hline
\end{tabular}

Fig. 3b Iterative Method: Coefficients of optimized regression models of gage outputs $R 1, R 2, \ldots, R 6$. 


\begin{tabular}{|c|c|c|c|c|}
\hline $\begin{array}{l}\text { MATH } \\
\text { TERM }\end{array}$ & $\begin{array}{l}\text { T-STATISTIC } \\
\text { (from Fig. 2a) }\end{array}$ & $\begin{array}{c}\text { EXACT SOLUTION } \\
\text { (uses coefficients of } \frac{\text { non-iterative method, }}{\text { see also Eq. (4), values given in Fig. } 2 \mathrm{~b})}\end{array}$ & $\begin{array}{c}\text { APPROXIMATION } \\
\text { (uses coefficients of } \frac{\text { iterative method, }}{\text { see also Eq. (9), values given in Fig. 3b) }}\end{array}$ & $\begin{array}{l}\text { STD. DEV. OF } \\
\text { LOAD RESID., \% }\end{array}$ \\
\hline INTERCEPT & +244 & $\eta_{0}=+5.650 E+01$ & $\frac{-a_{0}}{a_{1}}=+5.663 E+01$ & $0.0489 \%$ \\
\hline$R 1$ & +982 & $\eta_{1}=+2.915 E+00$ & $\frac{1}{a_{1}}=+2.909 E+00$ & $0.0566 \%$ \\
\hline$R 2$ & +29 & $\eta_{2}=+7.997 E-02$ & $\frac{-a_{2}}{a_{1} b_{2}}=+8.065 E-02$ & $0.0491 \%$ \\
\hline$R 3$ & +4 & $\eta_{3}=+2.409 E-03$ & $\frac{-a_{3}}{a_{1} c_{3}}=+3.105 E-03$ & $0.0492 \%$ \\
\hline$R 5$ & -11 & $\eta_{4}=-1.742 E-02$ & $\frac{-a_{4}}{a_{1} e_{4}}=-1.838 E-02$ & $0.0495 \%$ \\
\hline$|R 1|$ & -27 & $\eta_{5}=-2.561 E-02$ & $\frac{-a_{5}}{a_{1}\left|a_{1}\right|}=-2.444 E-02$ & $0.0492 \%$ \\
\hline$|R 2|$ & -18 & $\eta_{6}=-1.567 E-02$ & $\frac{-a_{6}}{a_{1}\left|b_{2}\right|}=-1.395 E-02$ & $0.0496 \%$ \\
\hline$|R 3|$ & +13 & $\eta_{7}=+8.287 E-03$ & $\frac{-a_{7}}{a_{1}\left|c_{3}\right|}=+7.948 E-03$ & $0.0490 \%$ \\
\hline$|R 5|$ & +14 & $\eta_{8}=+2.498 E-02$ & $\frac{-a_{8}}{a_{1}\left|e_{4}\right|}=+2.336 E-02$ & $0.0502 \%$ \\
\hline$R 5 \cdot R 5$ & -5 & $\eta_{9}=-8.925 E-06$ & $\frac{-a_{9}}{a_{1} e_{4} e_{4}}=-8.157 E-06$ & $0.0492 \%$ \\
\hline$R 1 \cdot|R 1|$ & -7 & $\eta_{10}=-3.293 E-05$ & $\frac{-a_{10}}{a_{1} a_{1}\left|a_{1}\right|}=-3.747 E-05$ & $0.0506 \%$ \\
\hline$R 2 \cdot|R 2|$ & -8 & $\eta_{11}=-3.187 E-05$ & $\frac{-a_{11}}{a_{1} b_{2}\left|b_{2}\right|}=-3.257 E-05$ & $0.0490 \%$ \\
\hline$R 5 \cdot|R 5|$ & +5 & $\eta_{12}=+8.664 E-06$ & $\frac{-a_{12}}{a_{1} e_{4}\left|e_{4}\right|}=+9.327 E-06$ & $0.0492 \%$ \\
\hline
\end{tabular}

Fig. 4 Comparison of exact solution with approximation of regression model coefficients of $N 1$. 